\title{
Results from the Direct Search for the Standard Model Higgs Boson at LEP
}

\author{
Jesus Marco* \\ Consejo Superior de Investigaciones Científicas, Instituto de Física de Cantabria, \\ Centro Mixto CSIC-Universidad de Cantabria, Santander, Spain \\ E-mail: 'Jesus. Marco@cern.ch'
}

ABstract: The direct search for the Standard Model Higgs boson at LEP, using $2.5 \mathrm{fb}^{-1}$ of $\mathrm{e}^{+} \mathrm{e}^{-}$collision data collected and analyzed by the four LEP collaborations, ALEPH, DELPHI, L3 and OPAL, at center-of-mass energies between 189 and $209 \mathrm{GeV}$, is presented. The combination of their results by the LEP Higgs Working Group sets a lower bound for the SM Higgs boson mass of $114.1 \mathrm{GeV} / c^{2}$ at the $95 \%$ confidence level. The excess observed in the higher mass zone, with a significance at the $2 \sigma$ level, is described.

\section{Introduction}

\subsection{The search for the SM Higgs boson at LEP200}

The LEP collider has been operated at CERN since August 1989 until November 2000, with four experiments, ALEPH, DELPHI, L3 and OPAL installed in the corresponding beam intersection points. The first phase from 1989 to 1995, LEP-1, operated at a center-of-mass collision energy around $91 \mathrm{GeV}$ with the objective of precisely measuring the parameters of the $\mathrm{Z}$ boson. In the second phase, LEP-2, the collision energy was increased from $130 \mathrm{GeV}$ up to a maximum of $209 \mathrm{GeV}$, and so was also known as LEP200. The main reason for this increase was the extension of the search range for the Higgs boson.

The objective in LEP200 was to "find the Higgs boson", but the first question is "which Higgs boson?". The initial answer is "the Standard Model Higgs boson", H, involved in electroweak simmetry breaking.

The second question, its mass range, is delimited by triviality and vacuum stability arguments. At LEP-1, from direct searches in the $\mathrm{Z} \rightarrow \mathrm{HZ}^{*}$ channel, a lower mass limit of $65 \mathrm{GeV} / c^{2}$ was established. Precision measurements of the electroweak parameters provide an indication of the preferred Higgs boson mass range, with a corresponding $95 \%$ confidence level upper bound of $\approx 200 \mathrm{GeV} / c^{2}$.

${ }^{*}$ Speaker. 
However the most interesting possibility comes from MSSM models, where the lightest Higgs boson, $h$, that would have similar production and decay modes to the SM Higgs boson, is expected in most "benchmarks" corresponding to different model parameters, to have a mass below $130 \mathrm{GeV} / c^{2}$.

\subsection{Production Channels and Backgrounds}

Higgs-strahlung ( $\mathrm{e}^{+} \mathrm{e}^{-} \rightarrow Z^{*} \rightarrow H Z$ ) is the main process for production of an SM Higgs boson at LEP200 energies, with a corresponding kinematical limit $m_{\mathrm{H}}<\sqrt{s}-m_{\mathrm{Z}}$. Fusion (WW) contribution, with no kinematical limit, is significative for very high masses.

The total integrated luminosity collected of more than $500 \mathrm{pb}^{-1}$ at $\sqrt{s}$ above $206 \mathrm{GeV}$ allows the exploration of a mass range up to the corresponding kinematical limit, 206-91 $115 \mathrm{GeV}$, where a cross section of around $50 \mathrm{fb}$ results on about 25 signal events expected.

As the SM Higgs boson is expected to decay mainly into a pair of $b$ quarks (the branching ratio for this decay mode is $80 \%$, to be compared ${ }^{1}$ with $7 \%$ branching ratio into $\tau \tau$ ), the final topologies expected at LEP-2 are the following:

- the four-jet channel, with the $\mathrm{Z}$ decaying into a pair of quarks and the Higgs boson into $b \bar{b}$, accounting for more than $50 \%$ of expected signal events, a channel requiring kinematics and $b$-tagging techniques to reduce the corresponding background.

- the channel with missing-energy, due to the presence of a pair of neutrinos, and with a pair of b jets from the Higgs decay; good energy-flow and hermeticity are the key together with $b$-tagging.

- the leptonic channels, with a pair of leptons from the $\mathrm{Z}$ boson decay and a pair of $b$ jets from the Higgs boson decay; the $\tau$ channel includes also the search for events where the Higgs boson would decay into a pair of $\tau$ leptons and the $\mathrm{Z}$ boson into quarks, giving a similar topology.

The main backgrounds are:

- WW background: when both W decay into quarks this is an important background in the four-jet channel, that can be reduced only requiring $b$-tag content in at least two of the jets; $W$ decays into a $\tau$ lepton, with a subsequent decay involving an energetic neutrino, contribute to the background in the missing-energy channel. The total cross section is $\approx 18 p b$.

- $\operatorname{QCD}\left(\mathrm{Z}^{0} \gamma\right)$ background: this $\mathrm{SM}$ process with a high cross section $(\approx 80 p b)$, is the source of events with gluon radiation either double or with splitting into $b \bar{b}$, that result in a four-jet topology; also double or single radiative events are a very important background in the missing energy channel.

\footnotetext{
${ }^{1} \mathrm{WW}^{*}$ decay mode starts to open only for high masses, with an expected branching ratio of $\approx 8 \%$ for $m_{\mathrm{H}}=115 \mathrm{GeV} / c^{2}$
} 
- ZZ background: this is the so-called irreducible background; with a cross section of $\approx 1 \mathrm{pb}$, and $30 \%$ of the cases with a $\mathrm{Z}$ boson decaying into a pair of b quarks, it is also the main reference in the search: visibility of the $\mathrm{ZZ}$ peak is a must to be confident on the Higgs boson search results.

The search for a $50 \mathrm{fb}$ signal, within a $100 \mathrm{pb}$ background was only possible thanks to several achievements: the excellent performance of the LEP accelerator; the use of advanced detection techniques, in particular silicon vertex detectors, and the improvement in the corresponding =tagging techniques; the kinematical reconstruction methods, and the use of multidimensional techniques in the analysis, combined with statistical methods to provide the final results.

\subsection{LEP performance}

LEP provided a very clean environment and an excellent performance: table'i $\overline{1}$ shows typical luminosities per experiment collected along the LEP-2 period, and table $\underset{2}{2}$ those relevant for the SM Higgs search, for each experiment.

\begin{tabular}{|l|cccccc|}
\hline year & $' 95$ & $' 96$ & $' 97$ & '98 & '99 & 2000 \\
\hline$\sqrt{s}(\mathrm{GeV})$ & $130-136$ & $161-172$ & 183 & 189 & $192-196-200-202$ & $204-205-206.5-208$ \\
\hline $\mathrm{L}\left(\mathrm{pb}^{-1}\right)$ & $3-3$ & $10-10$ & 60 & 170 & $30-80-80-40$ & $10-70-130-10$ \\
\hline
\end{tabular}

Table 1: Typical integrated luminosities per experiment collected at LEP-2.

\begin{tabular}{|l|cccc||c|}
\hline $\mathrm{L}\left(\mathrm{pb}^{-1}\right)$ & ALEPH & DELPHI & L3 & OPAL & LEP \\
\hline$\sqrt{s}>189 \mathrm{GeV}$ & 629 & 610 & 627 & 599 & 2465 \\
\hline$\sqrt{s}>206 \mathrm{GeV}$ & 130 & 142 & 139 & 130 & 542 \\
\hline
\end{tabular}

Table 2: Total integrated luminosities per experiment collected at center-of-mass energies relevant for the SM Higgs boson search at LEP-2.

\subsection{Experimental techniques: b-tagging}

$B$ hadrons fly a few millimeters after an $\mathrm{e}^{+} \mathrm{e}^{-}$collision at LEP, and their decay products have large impact parameters and transverse momentum compared to light quarks.

Silicon microvertex detectors, placed close to the beam spot, allow primary vertex reconstruction with a typical resolution below 30 microns, the measurement of the impact parameter respect to this primary vertex with a good resolution, typically in the range 10-200 microns, for $R \phi$ and $z$ coordinates, and secondary vertex reconstruction.

The b-tagging technique at LEP evolved from simple "offset-counting" to the assignement of a $b$-likelihood for each jet including the lifetime information from impact parameter measurement, but also taking into account the secondary vertex mass and decay length, the rapidity, and the transverse momentum of decay leptons.

Figure ${ }_{1}^{1} \overline{1}$, shows as an example the very nice performance of $b$-tagging in the OPAL experiment. Very high rejection against WW background requires a very good control of the tail, checked with the help of semileptonic events. 
OPAL
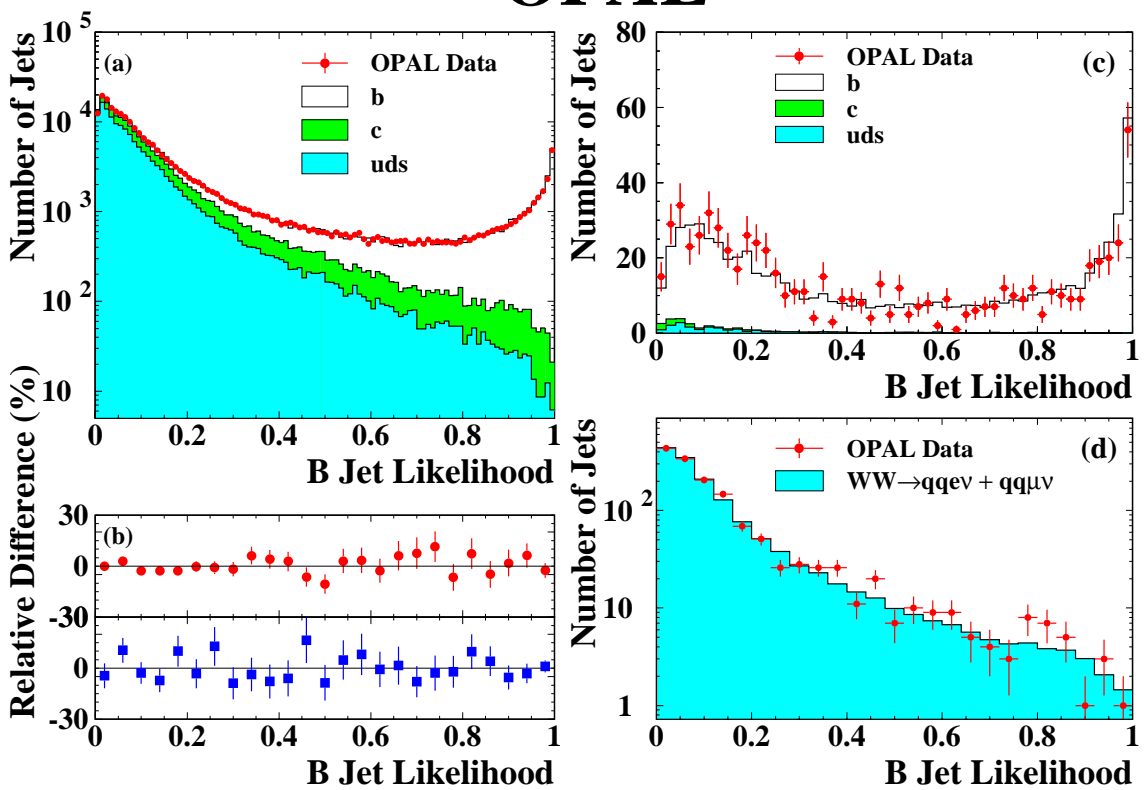

Figure 1: $b$-tagging performance and modelling for calibration (left-side plots) and high-energy data(right-side plots) in the year 2000 in OPAL.

\subsection{An analysis example: the four-jet channel in DELPHI}

As an example of the complexity of the developed analysis, a short description of the four-jet channel analysis in DELPHI is given below.

The analysis starts with a pre-selection, asking for "hadronic" events, not compatible with Initial State Radiation, and "spherical" in shape (not like two jets back-to-back).

Next step is the kinematical reconstruction: the DURHAM jet algorithm is used and energy and momentum conservation are imposed for 4-C and 5-C fits, essential to reach a resolution in the reconstructed Higgs mass of around $3 \mathrm{GeV} / c^{2}$.

Discriminant variables are then built against the different background sources:

- anti-QCD variables: "sphericity" variables, maximum dijet aperture cosmin, minimum dijet mass $\min \left(M_{i j}\right), E_{\min } \times \alpha_{\min }$, etc.

- anti-WW variables: event and di-jet $b$-tagging, $P_{W W}$ the kinematical compatibility with the WW hypothesis (from ideogram techniques).

- anti-ZZ variables: $P_{Z Z}$ the kinematical compatibility with the ZZ hypothesis.

All these variables are combined using neural network techniques into a single discriminating variable. 
Independently, the pairing for the mass reconstruction is done: the six possible configurations for jet assignment to the $\mathrm{Z}$ and Higgs dijets are considered, and for each one the result of a fit fixing the $\mathrm{Z}$ dijet mass to the nominal $\mathrm{Z}$ mass value, and the probability for the jet b-tagging values corresponding to the Higgs di-jet, are taken into account to build a likelihood used to select the adequate pairing. Only one single mass value is selected coming from the corresponding 5 - $\mathrm{C}$ fit.

The possible conflict in this unique mass assignment is visible in real data examples, like the event shown in figure $\sum_{i}^{-}$This event, not QCD-like, has two clear b-jets with a secondary vertex, and another two with intermediate $b$-tagging.

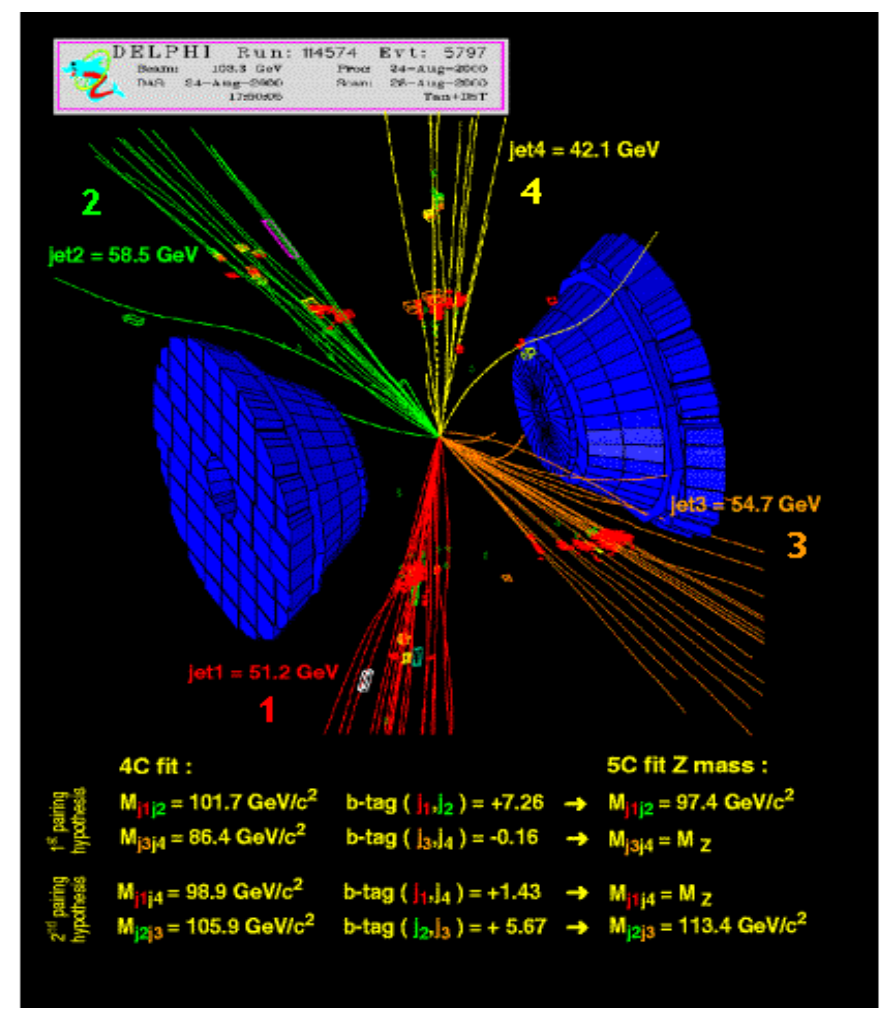

Figure 2: Graphical view of a relevant four-jet event collected by DELPHI.

Two hypothesis are possible: in the first one the selected configuration assigns the two clear $b$-jets to a dijet, and the other two to another dijet, and the reconstructed mass value is $97.4 \mathrm{GeV} / c^{2}$. In the second case, which would correspond to a four- $b$ event, the reconstructed mass would be $113.4 \mathrm{GeV} / c^{2}$, with an slightly better kinematical fit. The combined likelihood taking into account both the jets $b$-tagging values and the kinematical fit, selects the first hypothesis as the most likely, and so the unique assigned mass value is the lower one.

The two event variables, the output of the neural network and the reconstructed Higgs mass, are used in a 2-D computation of the signal-to-background ratio for a given Higgs mass hypothesis after carefully binning and smoothing required to avoid singularities and edge effects. 


\subsection{Confidence Level Estimation}

Estimation is derived from these sets of distributions ( $p d f \mathrm{~s}$ ) obtained from Monte Carlo simulated events for all backgrounds and the expected signal at a given Higgs mass value, and from the corresponding values for data events. The signal-to-background ratio assigned to each event allows a weighted counting method: in the classical counting (Poisson) method a subset of events was selected after a set of relevant cuts in the input variables assuring an optimal signal-background separation, and the estimator was defined simply by the number of events; in the weighted counting method the signal-to-background value for each event is used as a weight assigned to it, and the addition of all of them returns the final estimator, with no need for relevant cuts. The estimator used is the likelihood ratio [1] $-2 \ln Q=2 s_{t o t}-2 \sum_{i} N_{i} \ln \left[1+s_{i} / b_{i}\right]$ where $w_{i}=s_{i} / b_{i}$ are the signal-to-background ratios.

The confidence levels are estimated using a Monte Carlo method, where estimator distributions are built for the background only hypothesis and for the background plus signal hypothesis. Good separation between both distributions indicates a high sensitivity to the search. The discovery estimator, $1-C L_{B}$, computed as the integral in the previous distribution below the point marked by the estimator value observed in the data, reflects the departure from the Standard Model, while $C L_{S}$, conservatively defined as $C L_{S+B} / C L_{B}$ is used for the limit. Results in each different channel from the LEP experiments are combined by the LEP Higgs Working Group.

\section{The search for the SM Higgs in the year 2000}

\subsection{The starting point}

In the year 2000, the search started with a previous limit [2] of $108 \mathrm{GeV} / c^{2}$. By the July LEPC meeting at CERN, with around $50 \mathrm{pb}^{-1}$ collected at a center-of-mass energy of $205 \mathrm{GeV}$, and $25 \mathrm{pb}^{-1}$ at $206 \mathrm{GeV}$, per experiment, the combined limit reached $113.3 \mathrm{GeV} / c^{2}$, with no significative excess of events observed [i3] limit zone ("high mass" zone) was not excluded.

\subsection{The first significative candidates with high mass}

However, already by July LEPC, one significative candidate had been observed in the four-jet channel in ALEPH: with an assigned mass of $114 \mathrm{GeV} / c^{2}$, produced at a collision energy of $206.7 \mathrm{GeV}$, this event showed a $b b q q$ topology, and its high value in the cosmin variable (minimum sum of cosines of aperture dijet angles), -1.98 , and high $b$-tag, resulted in a signal-to-background value of $\approx 4$.7. Figure $\bar{\beta}_{-1}$ shows the graphical representation of this first significative event ${ }^{2}$.

Along August, and presented at the LEPC meeting of 5th of September [琣], two more strong four-jet candidates from ALEPH, both with a four- $b$ topology, were observed with reconstructed masses of $112.9 \mathrm{GeV} / c^{2}$ and $110 \mathrm{GeV} / c^{2}$; the only possible background for both of them is ZZ production, with either one of the $\mathrm{Z}$ slightly off-shell, or small undetected ISR, and wrong pairing assignment.

\footnotetext{
${ }^{2}$ more details can be found in the corresponding ALEPH publication [i
} 


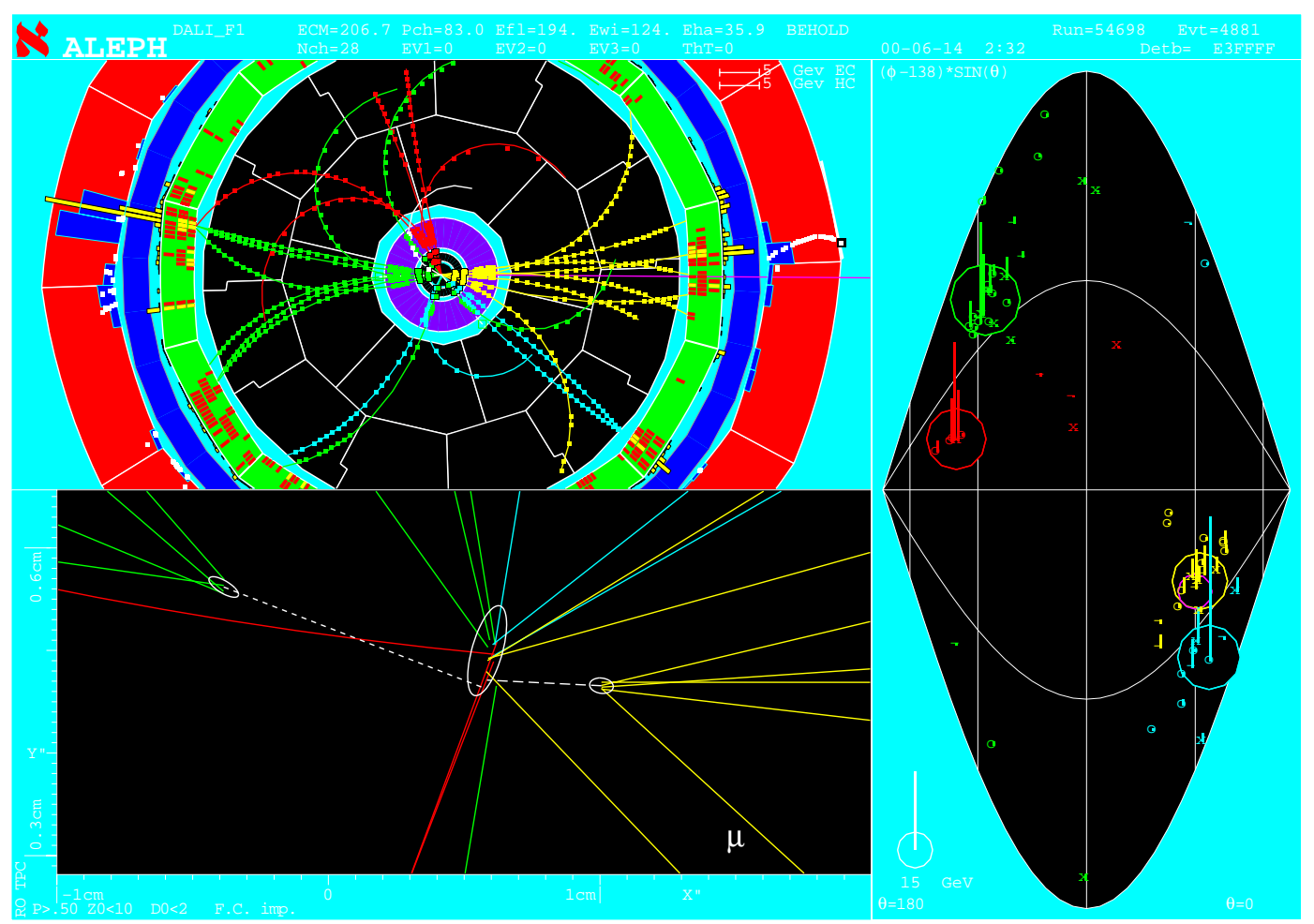

Figure 3: ALEPH four-jet candidate with $b b q q$ topology.

\subsection{Evolution of the excess at high mass}

A $3.9 \sigma$ excess observed by ALEPH for a mass hypothesis of around $114 \mathrm{GeV} / c^{2}$ was presented at the LEPC meeting of 5th of September, and reflected in the corresponding LEPHWG combination as a $2.2 \sigma$ excess, even if not supported by the results of any of the other LEP experiments.

Right after the LEPC meeting, DELPHI reevaluated their $s / b$ weights due to smoothing problems with the $p d f \mathrm{~s}$, and a four-jet candidate with a reconstructed mass close to $114 \mathrm{GeV} / c^{2}$ increased its $s / b$ due to a moderate b-tagging value originated by the presence of a reconstructed secondary vertex, even if kinematically it was compatible with the WW background hypothesis.

After an extra month of LEP operation, the next combination of results took place for the famous LEPC meeting of 3rd of November of 2000 [. At that time, the combination, with more than $490 \mathrm{pb}^{-1}$ collected at $\sqrt{s}>206 \mathrm{GeV}$ showed an excess at the $2.9 \sigma$ level; this evolution corresponded to the new results from each experiment : ALEPH had not found new significative candidates in the collected data; DELPHI after detector realignment had reduced the weight of the previous candidate since it lost its secondary vertex; OPAL had found no strong candidate but many ones contributing with low $s / b$ values, and the effect increase was due mainly to new candidates in L3, in the $H q q$ channel and specially in the missing-energy channel. In this channel a new candidate was observed with a reconstructed mass close to $114 \mathrm{GeV} / c^{2}$ and a corresponding $s / b$ value of around 2 . The event is shown in figure 岱. It has two well b-tagged jets, but the two fermion background is critical at the 
kinematical limit: double ISR events and $b b$ events (when the neutrinos take most of their energy) give collinear topologies similar to the one observed in this event. For an event at rest the mass recoiling to the $\mathrm{Z}$ is pushed to $\sqrt{s}-m_{\mathrm{Z}}$, while, due to the $\mathrm{Z}$ width, even in the Higgstrahlung close to the kinematical limit, the Higgs boson is not usually produced at rest.

Run \# 933204 Event \# 4704 Total Energy : $112 \mathrm{GeV}$
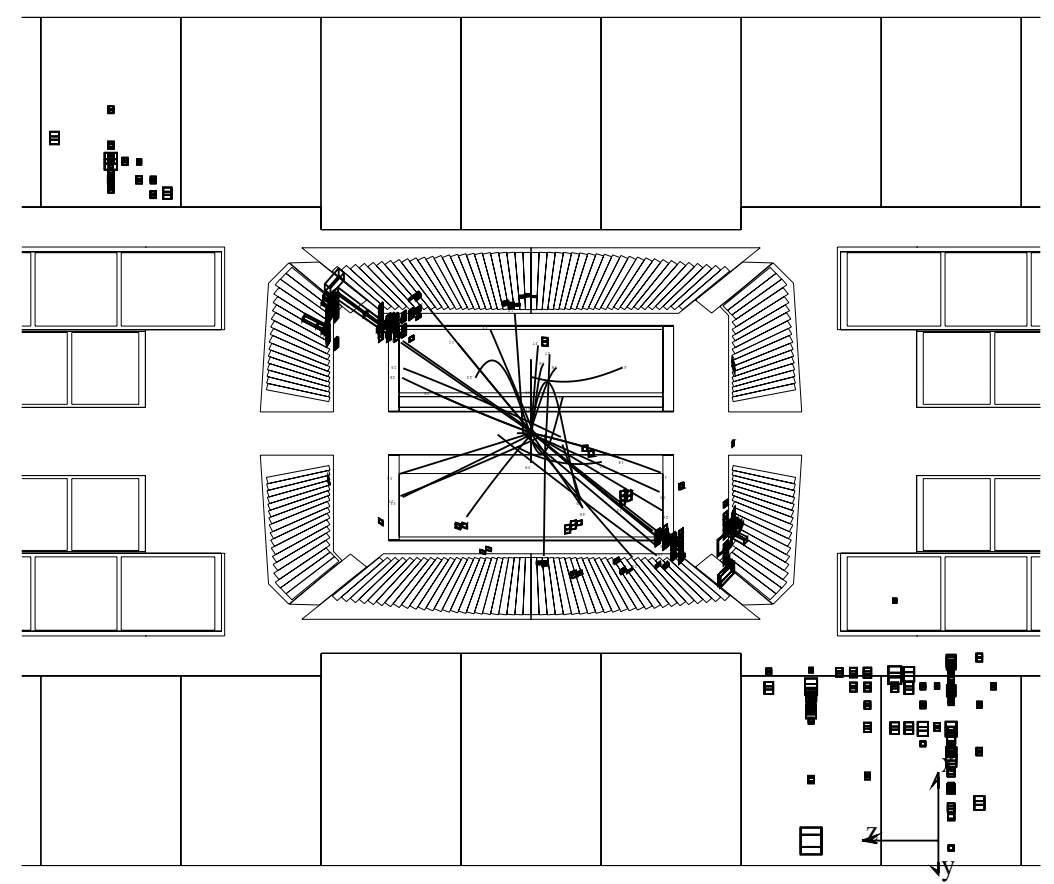

Figure 4: L3 missing energy candidate

At this LEPC meeting an extended running period for LEP in 2001 was requested, not granted by CERN management.

ALEPH [i, $\overline{6}]$ shortly published their result, "Observation of an Excess in the Search for the Standard Model Higgs Boson at ALEPH", and also L3 [8]" "Higgs Candidates in $e^{+} e^{-}$

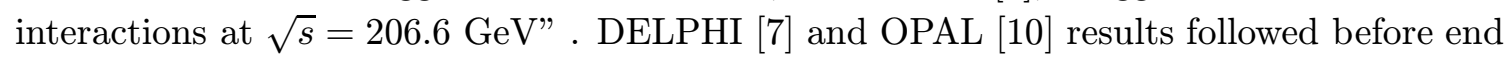
of the year.

Revised inputs with complete luminosities for the publication of the combined LEP Higgs Working Group result started to be prepared, while in parallel all collaborations aimed for final results. In July 2001 L3 released their final results in a new publication [ị], and a new set of inputs for combination. In these new results, with a more powerful $H q q$ analysis and a better background Monte Carlo simulation for the missing energy channel, the significance of previous candidates is reduced. 


\subsection{Current results: Summer 2001}

By summer 2001 the situation is as follows: ALEPH is finishing their final paper; DELPHI is finishing the final reprocessing of LEP200 data, with a significative improvement in the alignment, and producing very high Monte Carlo statistics; L3 has published their final results, and OPAL is working on the analysis optimization, aiming for a final paper before the end of the year.

The LEPHWG workshop in Evian (May 2001), discussed possible improvements before the final combination, expected by the end of the year, is prepared.

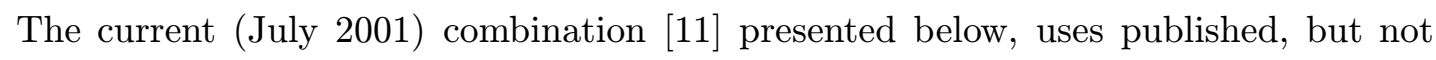
final results from ALEPH, DELPHI and OPAL, and final ones from L3.

The distributions of the reconstructed Higgs boson mass with increasing purity for a signal with mass $115 \mathrm{GeV} / c^{2}$ are shown in figure $\underline{\underline{5}}$.r.
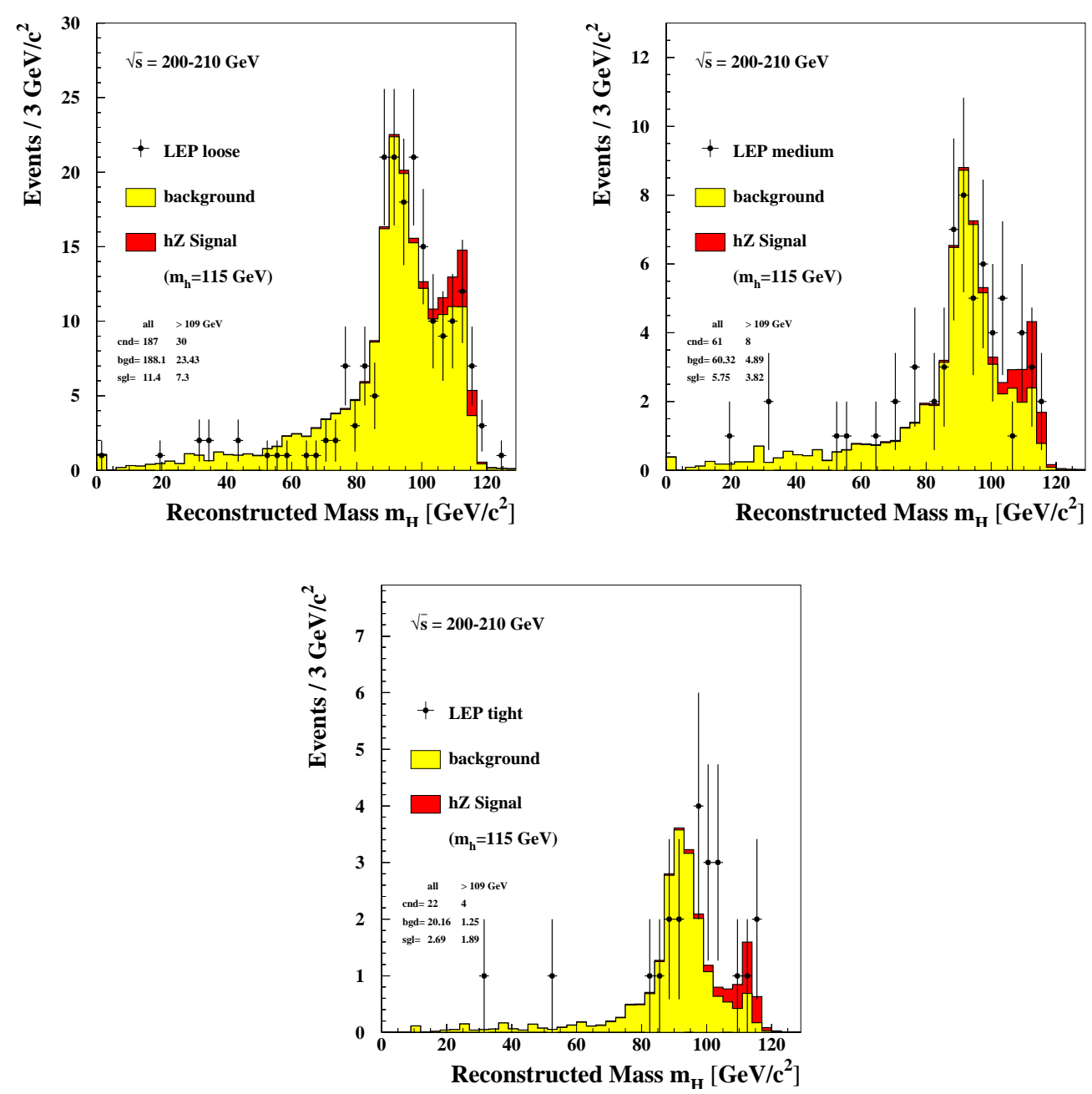

Figure 5: Distributions of the reconstructed Higgs boson mass, $m_{\mathrm{H}}$ at increasing level of purity for a signal with mass $115 \mathrm{GeV} / c^{2}$. 
The results in terms of the distribution of the test statistic as a function of the test

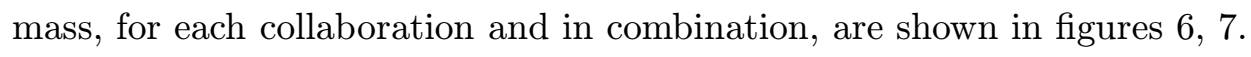
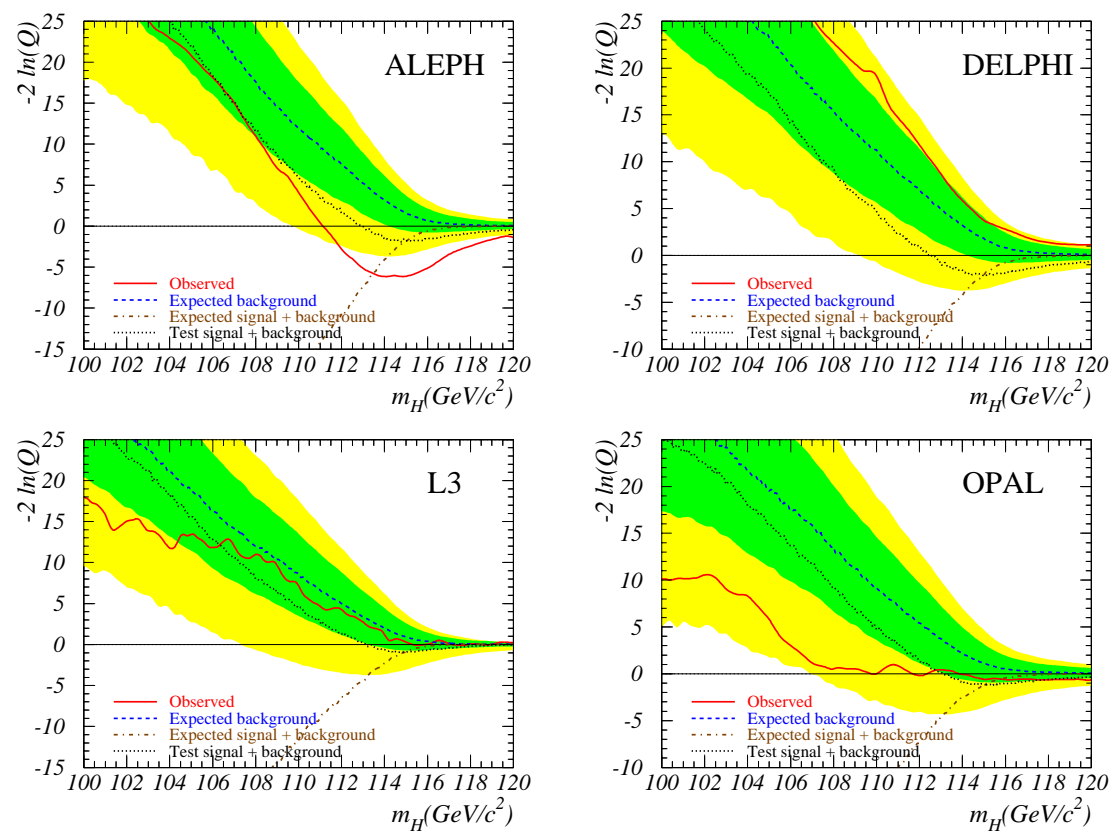

Figure 6: The test statistic $(-2 \ln Q)$ as a function of the test-mass $m_{\mathrm{H}}$ for each LEP experiment.

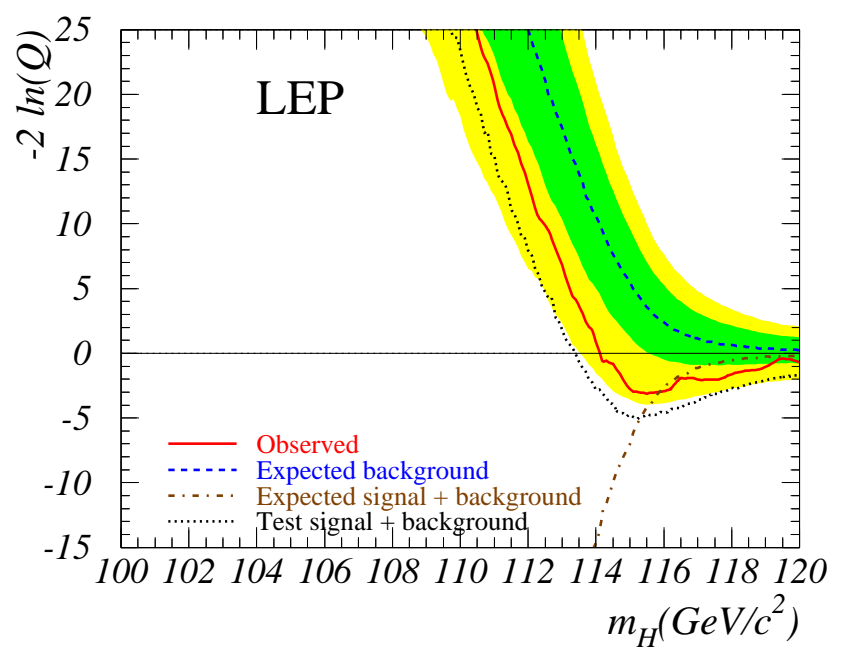

Figure 7: The test statistic $(-2 \ln Q)$ as a function of the test-mass $m_{\mathrm{H}}$, obtained from the combination. The actual observation is represented with a solid line while the dashed and dash-dotted lines represent the median for the background and signal+background hypothesis. Shaded bands indicate the \pm 1 and \pm 2 standard deviation respect to the background only hypothesis expectation. The dotted line indicates the result of a test including a fixed mass Higgs boson signal at $115 \mathrm{GeV} / c^{2}$. 
A lower limit on the Higgs mass of $114.1 \mathrm{GeV} / c^{2}$ is obtained from the confidence level for the signal+background hypothesis $\left(C L_{S}\right)$ curve $\bar{\beta}_{1}$, slightly below the expected value $\left(115.4 \mathrm{GeV} / c^{2}\right.$ ). Individual limits are $111.5 \mathrm{GeV} / c^{2}$ (expected $113.8 \mathrm{GeV} / c^{2}$ ) for ALEPH, 114.3 GeV/c $c^{2}\left(113.5 \mathrm{GeV} / c^{2}\right)$ for DELPHI, $112.2 \mathrm{GeV} / c^{2}\left(112.7 \mathrm{GeV} / c^{2}\right)$ for $\mathrm{L} 3$, and $109.4 \mathrm{GeV} / c^{2}\left(112.6 \mathrm{GeV} / c^{2}\right)$ for OPAL.

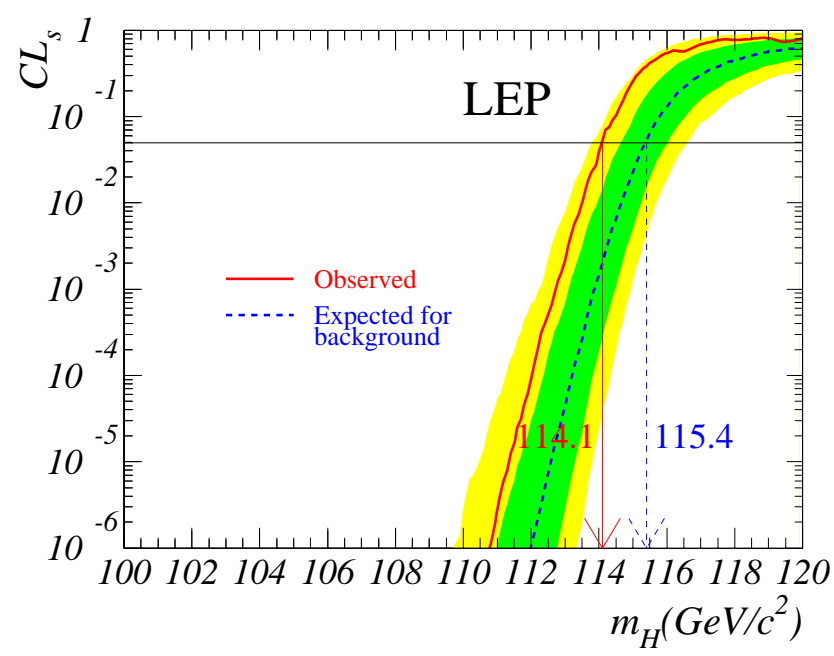

Figure 8: Confidence level $C L_{s}$ for the signal+background hypothesis.

\subsection{The excess at high mass}

The combined plot in'i shows a minimum at $115.6 \mathrm{GeV} / c^{2}$. The corresponding probability of a local fluctuation given by $1-C L_{b}$ is shown in figure $\bar{q}_{1}$ as a function of the test mass.

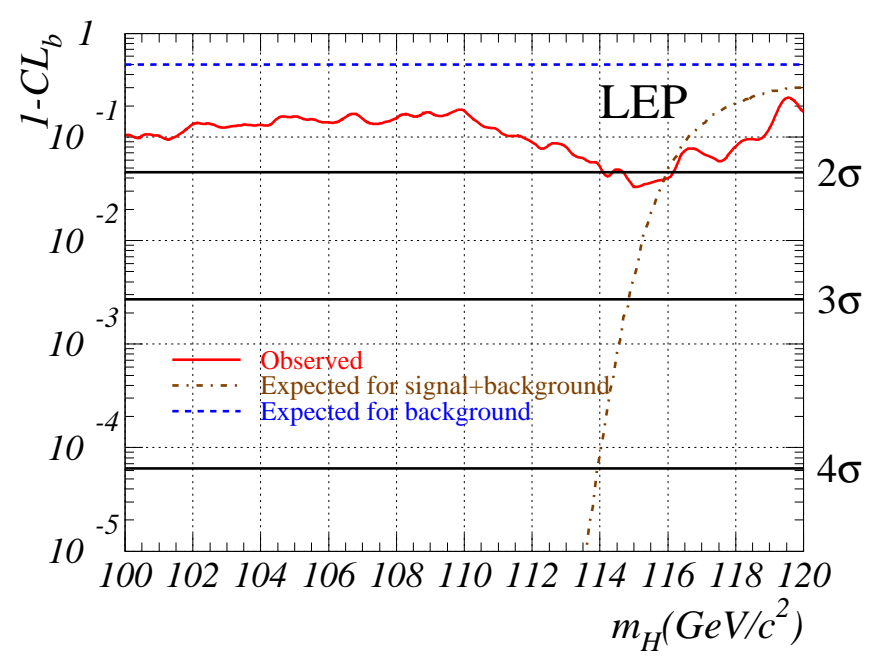

Figure 9: $1-C L_{b}$ as a function of the test mass: compatibility with the SM background. 
The combined LEP value for $1-C L_{b}$ for $\mathrm{mh}=115.6$ is 0.035 , at the $2 \sigma$ level ${ }^{3}$. The values corresponding to the individual results are 0.0023 for ALEPH, 0.88 for DELPHI, 0.25 for L3, and 0.22 for $O P A L$. The effect comes mainly from ALEPH, as was already visible in figure ${ }^{\prime} \overline{6} \cdot$. The combination of the other three experiments (DLO) results would return a value of 0.48 .

The likelihood ratio $-2 \ln Q$ is built up from individual event weights $w=\ln (1+s / b)$, and, as indicated before, few events are expected from a Higgs signal at the kinematical limit. The ten candidates with the highest weights for the hypothesis $m_{H}=115 \mathrm{GeV} / c^{2}$ are listed in Table $\overline{3}$, and the complete integrated event weight distribution is shown in figure $\bar{i} \underline{\underline{\alpha}}$.

\begin{tabular}{|l|c|c|c|c|c|c|}
\hline & EXP & $\sqrt{s}(\mathrm{GeV})$ & Channel & $M(\mathrm{GeV})$ & $s / b$ & $w$ \\
\hline \hline 1 & ALEPH & 206.7 & 4-jet & 114.3 & 4.6 & 1.73 \\
2 & ALEPH & 206.7 & 4-jet & 112.9 & 2.4 & 1.21 \\
\hline 3 & ALEPH & 206.5 & 4-jet & 110.0 & 0.9 & 0.64 \\
4 & L3 & 206.4 & E-miss & 115.0 & 0.7 & 0.53 \\
5 & OPAL & 206.6 & 4-jet & 110.7 & 0.7 & 0.53 \\
6 & DELPHI & 206.7 & 4-jet & 114.3 & 0.6 & 0.49 \\
7 & ALEPH & 205.0 & Lept & 118.1 & 0.6 & 0.47 \\
8 & ALEPH & 208.1 & Tau & 115.4 & 0.5 & 0.41 \\
9 & OPAL & 205.4 & 4-jet & 112.6 & 0.5 & 0.40 \\
10 & ALEPH & 206.5 & 4-jet & 114.5 & 0.5 & 0.40 \\
\hline
\end{tabular}

Table 3: List of the ten most significative candidates for the high mass hypothesis $m_{H}=$ $115 \mathrm{GeV} / c^{2}$. The experiment, collision energy, decay channel, reconstructed Higgs boson mass, $s / b$ ratio, and weight $(w)$ contributed to the test statistic are detailed. From Monte Carlo background simulation, 5.3 events are expected with $s / b$ above 0.5 .

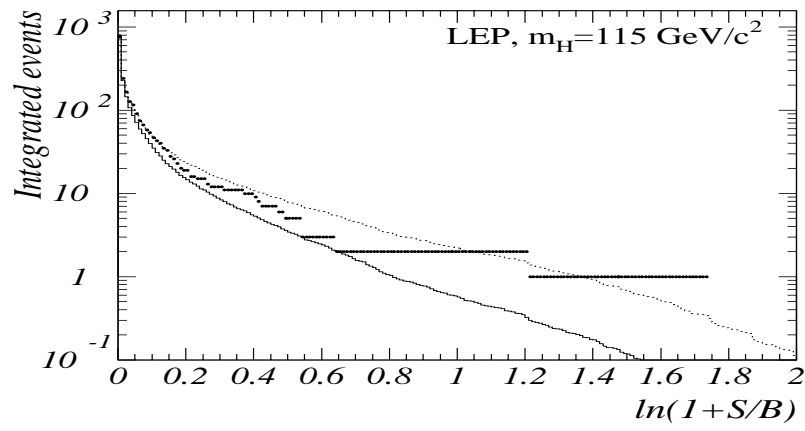

Figure 10: Integrated distribution of event weights for the mass hypothesis $m_{H}=115 \mathrm{GeV} / c^{2}$ : the discrete steps line corresponding to data can be compared to what expected from background only (lower curve) and signal plus background (upper curve).

\footnotetext{
${ }^{3}$ To obtain the probability for such a fluctuation to appear anywhere within a given mass range of interest, a multiplicative factor has to be applied, that can be estimated dividing the mass range (delimited by the previous exclusion limit, $108 \mathrm{GeV} / c^{2}$, and by the kinematic limit $\approx 116 \mathrm{GeV} / c^{2}$ ) by the average mass resolution $\left(\approx 3.5 \mathrm{GeV} / c^{2}\right)$.
} 
The contribution to the excess from the two most significative events, with $s / b>1$ is comparable to the contribution of all other events: the correct assignment of weight to individual highly significative events is critical, and requires a careful study of the corresponding background, including tails of $1 \mathrm{D}$ distributions and corners of $2 \mathrm{D}$ ones.

The combination by decay channel, figure most powerful one, is the main source of the final excess.
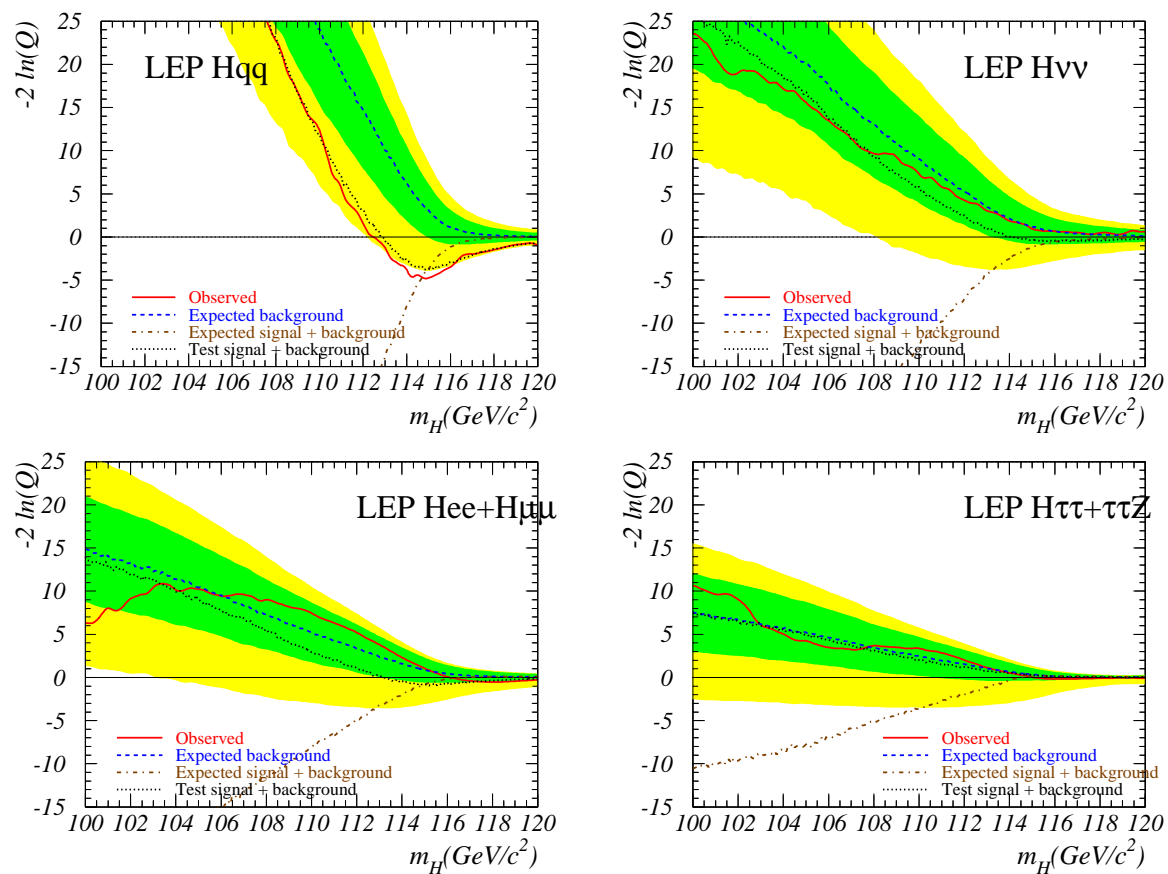

Figure 11: The test statistic $(-2 \ln Q)$ as a function of the test-mass $m_{\mathrm{H}}$ (see figure $\frac{\overline{1}}{1}$ ) for each search channel.

The evolution of the high mass excess from the LEPC results on November to the current ones can be summarized as a reduction of the $2.9 \sigma$ effect downto the $2 \sigma$ level, due to the following changes (including the use of the final complete sample, with $\approx 10 \%$ increase of the luminosity collected at $\sqrt{s}>206 \mathrm{GeV})$ : ALEPH has provided revised inputs and taken into account correlation effects, their background probability $\left(1-C L_{b}\right)$ for a Higgs boson mass hypothesis of $115 \mathrm{GeV} / c^{2}$ has changed from 0.00065 up to 0.0026 ; DELPHI has slightly improved the leptonic analysis, its $1-C L_{b}$ has changed from 0.68 to 0.77 ; L3, as said, has optimized their four-jet analysis and improved the missing-energy channel simulation, their background probability has increased from 0.068 to 0.32 ; and finally, OPAL has improved their background description, and $1-C L_{b}$ has changed from 0.19 to 0.20 .

Globally, the $2.9 \sigma$ effect is now reduced to a $2 \sigma$ effect, while the Higgs boson mass limit has increased from 113.5 to $114.1 \mathrm{GeV} / c^{2}$. 


\section{Conclusion}

Thanks to the excellent LEP performance, direct searches for the Standard Model-like Higgs boson have covered the mass range up to $115 \mathrm{GeV} / c^{2}$. All LEP collaborations have published results using 2000 data, and aim for final ones this year. The combination by the LEP Higgs Working Group gives a lower bound for $m_{\mathrm{H}}$ of $114.1 \mathrm{GeV} / c^{2}$ at 95\% C.L.

The excess observed in the high-mass zone (around $115 \mathrm{GeV} / c^{2}$ ) has decreased its significance to $2 \sigma$, and is mainly driven by the four-jet channel in ALEPH.

However, understanding the $m_{\mathrm{H}}=115 \mathrm{GeV} / c^{2}$ zone in the near future is very interesting but difficult, and one year more of LEP project would have been great!

\section{Acknowledgments}

I would like to thank my colleagues in the LEPHWG and in the DELPHI Higgs Research Line, for their help and interesting discussions while preparing this talk.

\section{References}

[1] A.L.Read in CERN Report 2000-005, p. 81 (2000).

[2] ALEPH, DELPHI, L3 and OPAL Collaborations, The LEP working group for Higgs boson searches, Searches for Higgs bosons: Preliminary combined results using LEP data collected at energies up to 202 GeV, CERN-EP/2000-055.

[3] ALEPH, DELPHI, L3 and OPAL Collaborations, The LEP working group for Higgs boson searches, Searches for Higgs bosons: Preliminary combined results using LEP data collected at energies up to $209 \mathrm{GeV}$, ALEPH 2000-074 CONF 2000-051, DELPHI 2000-148 CONF 447,L3 Note 2600, OPAL Technical Note TN661, submitted to ICHEP'2000, Osaka, Japan, July 27-August2, 2000.

[4] D. Schlatter for the ALEPH Collaboration, LEP Committee Open Session, 5.9.2000.

[5] P. Igo-Kemenes for the LEP Higgs working group, LEP Committee Open Session, 3.11.2000, http://lephiggs.web.cern.ch/LEPHIGGS/talks/index.html.

[6] ALEPH Collaboration, R. Barate et al., 'P

[7] DELPHI Collaboration, P. Abreu et al., 'P

[8] L3 Collaboration, M. Acciarri et al., Phys. Lett. B 495 (2000) 18.

[9] L3 Collaboration, P. Achard et al., 'Phys. Lett. B $517 \overline{1}(2001)$ 319.'.

[10] OPAL Collaboration, G. Abbiendi et al., PPhys. Lett. B $4999(2001) 38$

[11] ALEPH, DELPHI, L3 and OPAL Collaborations, The LEP working group for Higgs boson searches, Search for the Standard Model Higgs Boson at LEP, ALEPH 2001-066 CONF 2001-046, DELPHI 2001-113 CONF 536,L3 Note 2699, OPAL Technical Note PN479, contributed paper for EPS'01 in Budapest and LP'01 in Rome. 